\title{
Factors Associated with Severe Mucosal Injury of Esophagogastroduodenoscopy after Caustic Substance Ingestion in Pediatrics
}

\author{
Ma. Charina Batac-Dizon, ${ }^{1}$ Cynthia P. Cordero ${ }^{2}$ and Germana V. Gregorio ${ }^{1}$ \\ ${ }^{1}$ Section of Pediatric Gastroenterology, Hepatology and Nutrition. Department of Pediatrics, \\ College of Medicine and Philippine General Hospital, University of the Philippines Manila \\ 2Department of Clinical Epidemiology, College of Medicine, University of the Philippines Manila
}

\begin{abstract}
Objective. To determine the prevalence of and factors associated with severe mucosal injury on esophagogastroduodenoscopy (EGD) after caustic ingestion.

Methods. Consecutive patients $\leq 19$ years old with history of caustic ingestion were included. Factors considered were age, sex, caustic agent (acid or alkali) and presence of signs/symptoms (oral lesions, drooling, vomiting, abdominal pain, dysphagia).
\end{abstract}

Results. 320 patients were investigated: 155 (48\%) accidental and 165 (52\%) intentional cases. In accidental intake, majority (84\%) were $\leq 6$ years old [mean(SD) age: 3.7 (4.3) yrs, 59\% males]. $10 \%$ had severe mucosal injury. The odds of severe injury increased in the presence of $\geq 2$ signs/symptoms: $O R=7.0$ for 2 and $\mathrm{OR}=62.2$ for $>2$. In intentional cases, the mean (SD) age was 16 (1.6) years, 74\% females. Severe mucosal injury was seen in $5 \%$ and associated with acidic agent ( $\mathrm{OR}=54.8)$.

Conclusions. Severe mucosal injury on EGD occurred in 10\% and $5 \%$ among accidental and intentional cases, respectively. In accidental cases, probability of severe injury increased in the presence of $\geq 2$ signs/symptoms. In intentional cases, acid intake was the only factor associated with severe injury. In the local setting, presence of these factors among patients with caustic ingestion will help identify high-risk patients who need EGDguided management.

Key Words: acid, alkali, mucosal injury, Zargar, caustic ingestion

\section{Introduction}

Caustic substance ingestion is a common major health hazard in the pediatric age group. The resultant injury to the upper gastrointestinal tract mucosa may be insignificant but in some cases may lead to strictures or perforations. An

Corresponding author: Germana V. Gregorio, MD

Section of Pediatric Gastroenterology, Hepatology and Nutrition

Department of Pediatrics

Philippine General Hospital

University of the Philippines Manila

Taft Avenue, Ermita, Manila 1000 Philippines

Telephone: +6325269167

Fax No: +6325260150

Email: germana1@hotmail.com esophagogastroduodenoscopy (EGD), which is the exploration of the proximal gastrointestinal tract, ${ }^{1}$ is considered the most effective way in assessing the integrity of the mucosal damage after ingestion. However, published studies are contradictory and inconclusive as to whether routine EGD should be done in all patients with caustic substance ingestion as severe mucosal injury is only seen in $<30 \%$ of cases. Gaudreault ${ }^{2}$ concluded that signs and symptoms do not predict the presence and or the severity of esophageal damage and suggested that routine EGD be done in all patients. On the other hand, Crain ${ }^{3}$ and Betalli ${ }^{4}$ reported that the presence of two or more signs (vomiting, drooling, stridor) is a good predictor of esophageal injury. Other studies, all done in developed countries, have suggested that EGD is not indicated in asymptomatic patients with a recent history of accidental, but not for intentional ingestion. $5,6,7$

The present practice of the Section of Pediatric Gastroenterology of the University of the Philippines Manila-Philippine General Hospital is to do routine endoscopy on all cases of caustic ingestion regardless of symptoms. This is the recommendation of the Toxicology service of the hospital based on the premise that even in the absence of signs and symptoms, other factors like age of the patient, type of solution and nature of ingestion (whether accidental or intentional) play a role in mucosal injury. Silver jewelry cleaner, a substance with cyanide as the active component, and a $\mathrm{pH}$ of $9-10$ had also been demonstrated as a common caustic substance in our setting, but the exact mucosal damage caused by this solution remains unclear. However, it has been our experience that the majority of children with caustic ingestion only have normal or minimal lesions, especially if they are asymptomatic and the exposure is accidental. In 2004, an unpublished review of 52 Filipino children with caustic ingestion at the Philippine General Hospital identified sodium hypochlorite (a household bleaching agent) as the most common agent. Thirteen percent $(13 \%)$ of patients had severe mucosal injury and all of these patients were symptomatic. The study further reported that the probability of having severe mucosal injury was increased by six-fold in the presence of two or more symptoms. However, the sample size limited the conclusions and recommendations of the study. This study 
determined separately for accidental and intentional cases which among these factors (patient's age, gender, caustic agent), signs (oral lesions, drooling, stridor), and symptoms (vomiting, abdominal pain, dysphagia, hematemesis) are associated with severe mucosal injury on EGD after caustic substance ingestion. The identified factors can then guide clinicians in determining high risk patients who need EGDguided management. Application of our results to other settings should note that in our institution caustic ingestions of strong alkali (lye water, dishwasher granules) is not common since these are less accessible in the Philippines than in the Western world. The common alkali substances are sodium hypochlorite (household bleach) and silver jewelry cleaner.

\section{Methods}

\section{Study design, setting and study patients}

Consecutive patients $\leq 19$ years old with a history of caustic substance ingestion within 72 hours in duration were recruited at a tertiary hospital in Manila, Philippines from June 2006 to May 2009. Accidental and intentional cases were considered as two separate patient groups since the volume consumed and risk of damage were expected to be different. Patients who were seen 72 hours after ingestion, with minimal or questionable amount ingested and those who were clinically unstable to undergo an EGD were excluded.

\section{Study procedures and data collection}

After informed consent was obtained, a thorough history and physical examination were performed on the patients by a Pediatric Gastroenterology fellow. The nature of ingestion as to accidental or intentional was ascertained. Data on the following potential factors associated with severe mucosal damage were collected: age and sex of the patient, type of ingested caustic substance (acid or alkali) and the presence or absence of vomiting, abdominal pain, hematemesis and dysphagia. Patients were examined with emphasis on the following signs considered as potential factors: oral lesions, drooling of saliva and stridor. Induced vomiting was not considered a symptom. Dysphagia and abdominal pain were considered present only if the child was able to provide an objective description.

All EGDs were performed by a single Pediatric Gastroenterology fellow at the central endoscopy unit within 10 to 72 hours after caustic ingestion using the GIF N30 (Olympus America Inc, Melville, NY, USA) for patients less than $10 \mathrm{~kg}$ and GIF XQ40 or GIF-E (Olympus) in heavier children. To ensure proper visualization, uncooperative patients were provided with intravenous sedation or subjected to general anesthesia by a pediatric anesthesiologist. Topical pharyngeal application with $2 \%$ lidocaine was given to those without sedation.
The mucosal damage was assessed using Zargar's grading of caustic injury severity (Table 1). Grades 2B and 3 were classified as severe mucosal injury while Grades 1 and $2 \mathrm{~A}$ were judged as non-severe injuries. All patients were administered standard treatment for caustic substance ingestion after EGD. Those with grade 0 were discharged without treatment. Patients with Grades 1 and 2A were fed initially with clear liquids and subsequently given regular diet as tolerated. They were also advised to take a histamine receptor antagonist for two weeks. Patients with Grades 2B and 3 were placed on nothing per orem, given total parenteral nutrition and referred to pediatric surgery section for anticipatory care. A repeat EGD or an upper GI series, if possible, was also done on all patients with severe mucosal injury after 10-14 days.

Table 1. Zargar Grading of Caustic Injury Severity ${ }^{\dagger}$

\begin{tabular}{|c|c|}
\hline Grade & Appearance \\
\hline 0 & Normal \\
\hline $\begin{array}{c}1 \\
\text { (superficial) }\end{array}$ & Edema and hyperemia of mucosa \\
\hline $\begin{array}{c}2 \mathrm{a} \\
\text { (transmucosal) }\end{array}$ & $\begin{array}{l}\text { Hemorrhage, erosion, blisters, exudates or whitish } \\
\text { membranes, superficial ulcers }\end{array}$ \\
\hline $2 b$ & $\begin{array}{l}\text { Grade 2a plus deep discrete or circumferential } \\
\text { ulcerations }\end{array}$ \\
\hline $\begin{array}{c}3 \\
\text { (transmural) }\end{array}$ & $\begin{array}{l}\text { Deep ulceration, eschar formation with necrosis, full } \\
\text { thickness injury with and without perforation }\end{array}$ \\
\hline
\end{tabular}

\section{Ethical considerations}

This study underwent ethical and technical review by the Expanded Hospital Research Office of the Philippine General Hospital. Study commenced after approval was obtained. Only patients who gave their assent and whose guardians gave informed consent were included in the study.

\section{Data analysis}

The prevalence of severe mucosal injury was estimated. Univariate analysis followed by multiple logistic regression was used to determine which of the following potential factors (age, gender, caustic agent), signs and symptoms (as individual signs and symptoms and number of signs and symptoms categorized as $0,1,2,>2$ ) are associated with severe mucosal injury. Age was categorized as $\leq 6$ or $>6$ years old to focus on the preschool children who were more prone to accidental ingestion. For intentional cases, age was not grouped since there was only one patient in the $\leq 6$ year old category. All analyses were carried out using STATA 9 (Stata corporation, Texas USA), 95\% confidence level was used in all estimates. All hypothesis testing were done at $5 \%$ level of significance. 


\section{Results}

A total of 365 patients were seen during the study period. Forty five (45) patients were excluded for the following reasons: 24 did not consent to participate; 15 were referred $>72$ hours after ingestion; and six were clinically unstable and could not undergo EGD. All six unstable patients were intubated; five had intake of silver jewelry cleaner resulting in severe cyanide poisoning while the other patient presented as acute abdomen requiring immediate surgery. The remaining 320 patients underwent EGD within 10 to 72 hours (mean: 42 hours) after ingestion. One hundred fifty five $(155,48 \%)$ and $165(52 \%)$ patients had accidental and intentional intake, respectively.

\section{Accidental cases}

Socio-demographic, caustic agent and clinical profile (Table 2). The mean age of the 155 cases was 3.7 years (SD: 4.3), with majority $(84 \%) \leq 6$ years old. There were more males $(59 \%)$. Eighty eight percent ( $88 \%$ ) had alkali ingestion generally in the form of chlorine bleach (54) and silver jewelry cleaner (44). A total of 85 cases (55\%) were symptomatic. Oral lesion was the most common sign and vomiting the most common symptom. None presented with stridor and hematemesis.

Table 2. Characteristics of patients with accidental and intentional ingestion of caustic substance

\begin{tabular}{|c|c|c|c|c|}
\hline & \multicolumn{2}{|c|}{$\begin{array}{c}\text { Accidental } \\
n=155\end{array}$} & \multicolumn{2}{|c|}{$\begin{array}{c}\text { Intentional } \\
n=165\end{array}$} \\
\hline & Number & Percent & Number & Percent \\
\hline Sex, frequency of female & 63 & 40.7 & 122 & 73.9 \\
\hline Age $\leq 6$ years & 130 & 83.9 & 1 & 0.6 \\
\hline \multicolumn{5}{|l|}{ Ingested Substance } \\
\hline Acid & 19 & 12.3 & 13 & 7.9 \\
\hline Acetic Acid & 1 & & 1 & \\
\hline Boric Acid & 5 & & 0 & \\
\hline Hydrochloric Acid & 9 & & 9 & \\
\hline Oxalic Acid & 1 & & 1 & \\
\hline Phosphoric Acid & 1 & & 1 & \\
\hline Sulfuric Acid & 2 & & 1 & \\
\hline Alkali & 136 & 87.7 & 152 & 92.1 \\
\hline Potassium Hydroxide & 2 & & 1 & \\
\hline Sodium Carbonate & 7 & & 4 & \\
\hline Sodium hydroxide & 29 & & 6 & \\
\hline Chlorine bleach & 54 & & 43 & \\
\hline Silver jewelry cleaner & 44 & & 98 & \\
\hline \multicolumn{5}{|l|}{ Presenting sign } \\
\hline Oral lesions & 27 & 17.4 & 4 & 2.4 \\
\hline Drooling & 10 & 6.5 & 4 & 2.4 \\
\hline \multicolumn{5}{|l|}{ Presenting symptom } \\
\hline Vomiting & 68 & 43.9 & 93 & 56.4 \\
\hline Abdominal Pain & 24 & 15.5 & 49 & 29.7 \\
\hline Dysphagia & 9 & 5.8 & 17 & 10.3 \\
\hline \multicolumn{5}{|l|}{ Number of signs/symptoms } \\
\hline $3-4$ & 12 & 7.7 & 9 & 5.4 \\
\hline 2 & 27 & 17.4 & 36 & 21.8 \\
\hline 1 & 46 & 29.7 & 68 & 41.2 \\
\hline None & 70 & 45.2 & 52 & 31.5 \\
\hline
\end{tabular}

Endoscopic findings, patient management and outcomes. Severe mucosal injury was seen in 16 of the 155 cases; 53 had mucosal and transmucosal lesions and 86 patients had no mucosal injury on endoscopy. The prevalence of severe mucosal injury was $10 \%$ (95\% CI: 6.0-16.2). Of the 16 cases of severe mucosal injury, four had acid intake (acetic - 1, hydrochloric - 1 , phosphoric - 1 , and sulfuric - 1) and 12 with alkali (sodium hydroxide - 10, potassium hydroxide - 1 , and sodium carbonate - 1). Ten of these 16 patients were discharged after two weeks while six (3.8\%) had complications: strictures (5) and gastric perforation (1). Two patients died because of mediastinitis and aspiration pneumonia.

Factors associated with severe mucosal injury. Univariate analysis showed that all factors demonstrated trends of association with severe mucosal injury but some are not statistically significant (Table 3). Notable were female gender $(\mathrm{OR}=3.7)$, vomiting $(\mathrm{OR}=11.0)$, oral lesions $(\mathrm{OR}=12.0)$ and drooling $(\mathrm{OR}=20.2)$. The odds of severe injury increased as number of signs/symptoms increased (Table 4). Compared with asymptomatic patients, there was a six- and 68-fold increase in the presence of two and greater than two signs/symptoms, respectively.

Table 3. Univariate analysis of the potential factors associated with concurrent severe mucosal injury among patients with ACCIDENTAL caustic ingestion, $n=155$

\begin{tabular}{|c|c|c|}
\hline Characteristics & OR(95\% CI $)$ & P-value \\
\hline Age, $\leq 6$ years & $1.39(0.29-13.38)$ & 1.000 \\
\hline Female Gender & $3.68(1.10-14.17)$ & 0.029 \\
\hline Acidic Agent & $2.76(0.57-10.64)^{+}$ & 0.112 \\
\hline \multicolumn{3}{|l|}{ Symptoms } \\
\hline Vomiting & $11.02(2.35-102.24)$ & 0.000 \\
\hline Abdominal Pain & $1.98(0.42-7.40)$ & 0.277 \\
\hline Dysphagia & $2.69(0.25-16.01)$ & 0.234 \\
\hline \multicolumn{3}{|l|}{ Signs } \\
\hline Oral Lesions & $11.96(3.35-44.45)$ & 0.000 \\
\hline Drooling & $20.25(3.90-109.96)$ & 0.000 \\
\hline Number of Signs and Symptoms & & 0.000 \\
\hline 3-4 signs/symptoms & $68.00(10.71-431.91)$ & \\
\hline 2 signs/symptoms & $5.91(1.02-34.44)$ & \\
\hline 1 sign or symptom & $1.55(0.21-11.38)$ & \\
\hline
\end{tabular}

Table 4. Multiple logistic regression analysis of the potential factors of concurrent severe mucosal injury among patients with ACCIDENTAL caustic ingestion, $n=155$

\begin{tabular}{lcc}
\hline & Adjusted OR & \\
\hline Age, $\leq 6$ years & $2.93(0.44-19.56)$ & 0.266 \\
Female Gender & $3.17(0.82-12.27)$ & 0.094 \\
Acidic Agent & $1.89(0.40-8.86)$ & 0.421 \\
Number of signs/symptoms & & \\
One & $1.53(0.20-11.54)$ & 0.682 \\
Two & $6.99(1.12-43.67)$ & 0.037 \\
Three to Four & $62.20(9.14-423.46)$ & 0.000 \\
\hline
\end{tabular}

\section{Intentional cases}

Socio-demographic, caustic agent and clinical profile (Table 2). The mean (SD) age was 16.2 (1.6) years with the youngest patient at six years and oldest at 18 years old. Majority $(74 \%)$ were females. The most common agent (59\%) was silver 
jewelry cleaner. Of the 165 cases, 113 (68\%) were symptomatic. Vomiting and abdominal pain were commonly seen. Only four patients had oral lesions, two of these also had drooling. Two additional patients were positive for drooling. None presented with stridor and hematemesis.

Endoscopic findings, patient management and outcomes. Of the 165 intentional cases, severe mucosal injury was seen in eight cases, with a prevalence of $4.8 \%$ (95\% CI: 2.1-9.3). Sixty four $(64,39 \%)$ patients had mucosal and transmucosal lesions and 93 (56\%) had no mucosal injury. Of the eight cases of severe mucosal injury, six had intake of acid (hydrochloric acid [muriatic acid] in five, oxalic acid in one) and two with alkali (silver jewelry cleaner and sodium hydroxide). Seven of the eight cases were symptomatic. Four were sent home after two weeks as the repeat EGDs were normal, including the asymptomatic 16-year-old patient who had intentional intake of silver jewelry cleaner. The other four had morbidities: esophageal strictures (3) and gastric outlet obstruction (1).

Factors associated with severe mucosal injury. Among those with intentional intake, univariate analysis showed an increased risk of severe mucosal injury with acidic agent ingestion $(\mathrm{OR}=64.3)$ (Table 5). Excluding cases who took silver jewelry cleaner, the OR was reduced to 45.4. All signs and symptoms showed trends of increased odds of severe injury but only drooling ( $\mathrm{OR}=25.8)$ is statistically significant. The odds were also increased with two or more signs or symptoms. Multiple logistic regression analysis showed that acidic ingestion was the only factor associated with severe mucosal injury (Table 6).

Table 5. Univariate analysis of the potential factors of severe mucosal injury among patients with INTENTIONAL caustic ingestion, $n=165$

\begin{tabular}{lcc}
\hline \multicolumn{1}{c}{ Characteristics } & OR $(\mathbf{9 5} \% \mathbf{C I})$ & p-value \\
\hline Female Gender & $0.57(0.11-3.85)$ & 0.431 \\
Acid Agent & $64.28(8.73-698.66)$ & 0.000 \\
Symptoms & $5.78(0.71-264.01)$ & 0.140 \\
$\quad$ Vomiting & $1.45(0.22-7.78)$ & 0.696 \\
$\quad$ Abdominal Pain & $3.16(0.28-19.62)$ & 0.193 \\
$\quad$ Dysphagia & & \\
Signs & $7.33(0.12-103.34)$ & 0.182 \\
$\quad$ Oral Lesions & $25.83(1.52-387.04)$ & 0.012 \\
$\quad$ Drooling & & \\
Number of signs and symptoms & $14.57(1.16-182.39)$ & 0.028 \\
3-4 signs/symptoms & $6.38(0.68-59.61)$ & \\
2 signs/symptoms & $0.76(0.05-12.46)$ & \\
1 sign or symptom &
\end{tabular}

Table 6. Multiple logistic regression analysis of the potential factors of severe mucosal injury among patients with INTENTIONAL caustic ingestion, $\mathrm{n}=165$

\begin{tabular}{lcc}
\hline \multicolumn{1}{c}{ Characteristics } & $\begin{array}{c}\text { Adjusted OR } \\
(\mathbf{9 5 \%} \text { CI) }\end{array}$ & p-value \\
\hline Age (in years) & $1.20(0.57-2.52)$ & 0.637 \\
Female Gender & $0.60(0.08-4.47)$ & 0.617 \\
Acid Agent & $54.77(7.58-395.57)$ & 0.000 \\
Number of signs and symptoms & & \\
One & $0.52(0.02-11.58)$ & 0.679 \\
Two & $6.13(0.41-92.12)$ & 0.190 \\
Three to Four & $3.11(0.12-77.49)$ & 0.489 \\
\hline
\end{tabular}

\section{Discussion}

At present, there is no consensus statement on whether routine esophagogastroduodenoscopy (EGD) should be done on all patients with caustic substance ingestion. This is because most studies were inconclusive due to small sample size, or used retrospective data collection so that the EGDs were done by different endoscopists. Our study recruited a large nu mber of children in a tertiary hospital in the Philippines with either accidental or intentional ingestion and a standard procedure was used in the assessment of patients, with only one endoscopist doing the EGDs on all patients.

Our study demonstrated that in the accidental cases, $10 \%$ had severe mucosal injury. There is an increased risk of severe mucosal injury on EGD in the presence of two or more signs and symptoms, with oral lesions and vomiting identified as more important findings based on univariate analysis. Our result is in contrast with that of Gaudreault ${ }^{2}$ from North America who reported that signs and symptoms do not predict esophageal injury but confirmed reports by Betalli ${ }^{4}$ and Lamireau ${ }^{8}$ who both recommended that EGD is only warranted in symptomatic patients. The report of Gaudreault ${ }^{2}$ was a retrospective study; thus, inter-observer variability might have occurred between different physicians who assessed and performed the endoscopy. In contrast, standard assessment of mucosal injury was ensured in our study and Lamireau's. ${ }^{8}$ Similar to the results of Betalli' ${ }^{4}$ and Lamireau's, ${ }^{8}$ the majority of our patients with severe mucosal injury also had intake of strong acid $(\mathrm{pH}<2)$ and strong alkali $(\mathrm{pH}>12)$.

Among cases with intentional intake, only $5 \%$ of our patients had severe mucosal injury with only four of 165 (3\%) patients developing complications. This is in contrast to the higher incidence (30 to 36\%) of severe esophageal injury and complication rate (30\%) reported in adults. ${ }^{9,10}$ This may be because majority of the cases in the current study are adolescents and most of them do not really intend to commit 
suicide but ingest the substance because of trivial reasons. The slower rate of ingestion and the smaller amount of substance ingested may not be enough to cause severe mucosal injury. Among the factors considered, acid intake showed an increased risk of severe mucosal injury. These acidic products are usually household cleaning substances like hydrochloric and oxalic acids. This confirms a previous adult study that showed association of acid intake, ${ }^{11}$ particularly glacial acetic acid with higher frequency of complications. The esophagus may be spared after acid ingestion due to rapid transit and the resistance of squamous epithelium but the ingestion produces pylorospasm and antral mucosal edema, inflammation and pyloric stenosis secondary to extensive fibrosis. Similar to previous reports, ${ }^{4,10-12}$ both esophageal stricture and gastric outlet obstruction $^{12,13}$ were observed in our series of patients with acid intake. Our findings suggest that patients with intentional ingestion of silver jewelry cleaner, a substance with a $\mathrm{pH}$ of 9-10 and known to have minimal effect on the mucosa, do not really warrant routine EGD especially if they are asymptomatic. Similarly, none of the 43 patients with intentional ingestion of household bleach developed severe mucosal damage. Commercial household bleaches in the Philippines containing sodium hypochlorite are agents that may have similar effects as silver jewelry cleaner with a $\mathrm{pH}$ of 8-9, acting as mucosal irritants, emetics and weak corrosives.

Esophagogastroduodenoscopy is a safe procedure in experienced hands although the procedure may require sedation in uncooperative patients. In our setting, all patients below 10 years old are intubated and placed on general anesthesia. A study by Thakkar ${ }^{14}$ reported a $2 \%$ higher complication rate in pediatric as compared to adult upper endoscopy. Among these complications, 66\% were due to reversible hypoxia, likely related to sedation while $12 \%$ were secondary to gastrointestinal bleeding. The procedure is also costly and compounds the anxiety of both parents and patients. The results of our study therefore call for a re-evaluation of our current practice of doing routine endoscopy on all cases of caustic substance ingestion. Asymptomatic patients should first be observed and routine EGD should only be done among those with a high risk of developing severe mucosal injury.

Our study is not without its limitations. We did not consider the exact quantity, dilution and $\mathrm{pH}$ of ingested agent. This would have been helpful in further categorizing the ingested substance. We recommend that these, as well as other factors like a product's physical state whether taken as a liquid or a solid form, viscosity and concentration of the solution be considered in future investigations. Moreover, although the grading of caustic injury was based on objective findings, the timing of EGDs were different, thus mucosal damage might have been missed during early endoscopy. It should also be noted that in children, symptoms of abdominal pain and dysphagia may be due to other reasons like hunger and induced vomiting rather than secondary to th e caustic ingestion. Our results could not be generalized for all children with caustic ingestion as factors associated with severe injury are expected to vary according to local experience.

In conclusion, in accidental cases, $10 \%$ had severe mucosal injury and the presence of two or more signs and symptoms was associated with severe mucosal injury on EGD. In intentional cases, only $5 \%$ had severe injury and intake of acid agent was the only factor identified to be associated with severe mucosal injury. In our setting, the presence of these factors among patients with caustic substance ingestion will help identify high-risk patients who need EGD-guided management.

\section{References}

1. Wilsey MJ Jr, Scheimann AO, Gilger MA. The role of upper gastrointestinal endoscopy in the diagnosis and treatment of caustic ingestion esophageal strictures, and achalasia in children. Gastrointest Endosc Clin N Am. 2001; 11(4):767-87.

2. Gaudreault P, Parent M, McGuigan MA, Chicoine L, Lovejoy FH Jr. Predictability of esophageal injury from signs and symptoms: a study of caustic ingestion in 378 children. Pediatrics. 1983; 71(5):767-70.

3. Crain EF, Gershel JC, Mezey AP. Caustic ingestions: symptoms as predictors of esophageal injury. Am J Dis Child. 1984; 138(9):863-5.

4. Betalli P, Falchetti D, Giuliani S, et al. Caustic ingestion in children: is endoscopy always indicated? The results of an Italian multicenter observational study. Gastrointest Endosc. 2008; 68(3):434-9.

5. Gorman RL, Khin-Maung-Gyi MT, Klein-Schwartz W, et al. Initial symptoms as predictors symptoms as predictors of esophageal injury in alkaline corrosive ingestions. Am J Emerg Med. 1992; 10(3):189-94.

6. Nuutinen M, Uhari M, Karvali T, Kouvalainen K. Consequences of caustic ingestions in children. Acta Paediatr. 1994; 83(11):1200-5.

7. Christesen HB. Prediction of complications following unintentional caustic ingestion in children: is endoscopy always necessary? Acta Paediatr. 1995; 84(10):1177-82.

8. Lamireau T, Rebouissoux, Denis D, Lancelin F, Vergnes P, Fayon M. Accidental caustic ingestion in children: Is endoscopy always mandatory? J Pediatr Gastroenterol Nutr. 2001; 33(1):81-4.

9. Cheng HT, Cheng CL, Lin CH, et al. Caustic ingestion in adults. The role of endoscopic classification in predicting outcome. BMC Gastroenterol. 2008; 8:31.

10. Arévalo-Silva C, Eliashar R, Wohlgelernter J, Elidan J, Gross M. Ingestion of caustic substances: a 15-year experience. Laryngoscope. 2006; 116(8):1422-6.

11. Poley JW, Steyerberg EW, Kuipers EJ, et al. Ingestion of acid and alkaline agents: outcome and prognostic value of early endoscopy. Gastrointest Endosc. 2004; 60(3):372-7.

12. Baskin D, Urganci N, Abbasoglu L, et al. A standardized protocol for the acute management of corrosive ingestion in children. Pediatr Surg Int. 2004; 20(11-12):824-8.

13. Wason S. The emergency management of caustic ingestions. J Emerg Med. 1985; 2(3):175-82.

14. Thakkar K. El Serag HB, Mattek N, Gilger MA. Complications of pediatric EGD: a 4 year experience in PEDS-CORI. Gastroinntest Endosc. 2007; 65(2):213-21. 